\section{Torgny Greitz, MD, PhD, FACR, Professor of Neuroradiology, Emeritus}

T orgny Vilhelm Bernhard Greitz, MD, Professor at the Karolinska Institutet, distinguished leader and innovator of neuroradiology, died August 1, 2016. He was trained in the Stockholm School of Neuroradiology at the Serafimer Hospital, where neurosurgery, headed by Herbert Olivecrona, and neuroradiology at the helm of Erik Lysholm were developed in close cooperation during the 1930s and 1940s. In his capacity as chief of the Department of Neuroradiology at the Karolinska Hospital, he has conveyed to new generations the know-how of those days with great astuteness. In the early digital era, he was a front figure in the development of clinical applications of CT and positron-emission tomography.

Torgny Greitz's influence on North American neuroradiology was considerable. On September 25, 1956, he arrived at Washington University in St. Louis to spend a year practicing and teaching neuroradiology at the Mallinckrodt Institute of Radiology. His visit had been arranged by Lysholm's successor Erik Lindgren, Torgny's teacher at the Serafimer Hospital, who, the year before, had made a lecturing tour to many of the important radiology institutions in the United States. Torgny Greitz's time at the Mallinckrodt Institute of Radiology would make a lasting impression. He introduced the daily routine used at the Serafimer Hospital, which imposed a much more active role for the radiologist than what was the practice in the United States. The radiologist should not merely order a set of x-ray exposures from a radiographer, all according to a fixed program, and afterward file a report but should rather decide the method and the imaging protocol to be used in each case and be busy in the x-ray room; make punctures of arteries or the spinal theca if so needed with the overall objective to establish the anatomic and physiologic state of the patient; and suggest the most probable diagnosis. Torgny proved for anyone to see, including skeptical neurosurgeons, that a trained neuroradiologist was able to perform the then often invasive procedures safely and interpret them accurately. Hugh Wilson, head of radiology in MR imaging, was impressed by Torgny's achievements and encouraged by him; Dr Wilson established a neuroradiology section at the Mallinckrodt Institute of Radiology, which quickly became a role model in the subspecialty. This would inspire other centers to follow. Juan Taveras at the Neuroradiological Institute and Manny Schechter at the Albert Einstein Medical Center, both in New York, started training programs in neuroradiology the same year (1956). At the Silver Jubilee of the American Society of Neuroradiology in 1987, Torgny Greitz from the Stockholm School got recognition for his contributions during the early days of North American neuroradiology.

Torgny Greitz was born in Stockholm on August 13, 1921, the eldest of 3 brothers. His father, Bernhard Greitz, was a media personality, busy, in turn, as a journalist at a daily radical newspaper, as editor of a cultural magazine dedicated to modern literature and poetry, and as culture attaché in Copenhagen. Torgny's mother, Vivi Ramberg, was a bank official, who met her future husband when breaking a leg skiing in Bernhard's company.

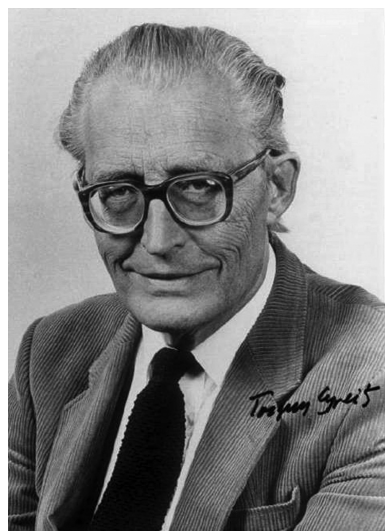

\section{School}

The family's home was welcoming to a broad circle of friends, many of whom had cultural aspirations. In his memoirs, left unpublished to posterity, Torgny paints a vivid picture of his early life. At 3 years of age, he was so absent-minded that his parents gave him the nickname "the professor." At school, he was soon appreciated and encouraged by his teachers. He became competitive and aspired eagerly to get the better of his classmates. He had an almost uncanny gift for memorizing. He learned his lessons in minimal time and developed an appetite for practical jokes. He describes himself as a mischievous little rascal. In the upper grades at school and continuing during his university years, he devoted himself to a wealth of activities: fine arts like drawing, painting, sculpting, but also athletics like track and field sports, even wrestling and swimming. With his competitive mind, he usually proved to be much above average in most of these activities. $\mathrm{He}$ once won the student championship in the decathlon when he managed $3.30 \mathrm{~m}$ in the pole vault, at a time when the pole still was made of bamboo (in 1940, the Swedish record was $4.18 \mathrm{~m}$ ).

Although a talented amateur painter and sculptor, he gave up early thoughts of devoting himself to the arts for a living. A decisive moment was when he read a book, Microbe Hunters by Paul de Kruif, about the great pioneers in medicine, among others Paul Ehrlich, Robert Koch, and Louis Pasteur. He was fascinated by the stories about how these researchers with their ingenuity and primitive tools made their revolutionary discoveries. Encouraged by his uncle, Johannes Ramberg, a medical doctor, and last but not least by Erik Lysholm, who was a friend of his parents, he decided to become a physician and devote his life to medical research.

\section{University}

He entered medical school at the Karolinska Institutet in 1940, incidentally in the same class as a future life-long friend of his, Sven-Ivar Seldinger, another Swede to become a great innovator of radiology. With cheerful temper, Torgny participated enthusiastically in the amusements of happy student life. An episode that made him and his study companion Lars Gyllensten already renowned when medical students is worth recalling. During a binge on beer and sandwiches, Torgny, on command from his friend, pronounced single words he picked at random from his imagina- 
tion or from objects in the room around him. His friend put them together in unrhymed and rather, but not altogether, unintelligible poems. One short example in translation (by his friend Giovanni Ruggiero, a neuroradiologist) reads, "The greatest sin/is that of the orphans/the snotty ones/who never had a nickel marble/the greatest sin/is to be nobody's darling." They managed to have their "oeuvre" published as written by an unknown debutant, Jan Victor, a pen-name. The reviews were benevolent. After a couple of days, the "authors" disclosed the truth. The news was soon on the first page in the daily papers all over Sweden. With a practical joke, 2 medical students had challenged the genuineness of the flourishing modern lyrics of the 1940s — or had they? A debate followed on the culture pages but soon calmed down. Torgny's accomplice, Lars Gyllensten, later had a brilliant career as an author, and Torgny Greitz, endowed with a linguistic vein and a sharp pen, would, late in his life, be awarded a prize for "his efforts to promote good medical language" by the Swedish Medical Society.

Torgny married Greta Schölin, a physiotherapist, the year before he finished medical school in 1948 . Soon the family also included 3 sons.

\section{Clinical Career}

After medical school, Torgny did 2 years' residency in surgery (Stockholm South Hospital and the Serafimer Hospital). His career in radiology was straight as an arrow and started at Stockholm South Hospital as a resident in 1950-53, followed by a position as radiologist at the Serafimer Hospital in 1954-58. After a feat of great strength and persistence during the summer of 1956, he was able to defend his seminal thesis, "A Radiologic Study of the Brain Circulation by Rapid Serial Angiography of the Carotid Artery." This happened only 3 days before he left for St. Louis.

In 1960, at 38 years of age, Torgny accepted a position as professor of general radiology at the Medical College of Umeå in the North of Sweden, where a new university was being established. He maintained this position until the neuroscience departments at the Serafimer Hospital were moved to a modern building at the Karolinska Hospital in 1963. Torgny Greitz was the indisputable choice for the position as head of an independent Department of Neuroradiology. He would stay in this position until his retirement in 1987. The same year as the digital image revolution was about to start, 1973, Torgny was appointed Professor of Neuroradiology, which rendered academic recognition to the discipline of neuroradiology. He reached the height of his career as President of the XIII Symposium Neuroradiologicum in Stockholm in 1986.

\section{Tutoring}

When Torgny set out to work at the Karolinska Hospital in 1963, his reputation as a dynamic innovator was recognized far and wide. Despite scarce staffing, he very soon managed to transform the department into a cutting-edge research center. Students from both North America (Hans Newton among others) and European countries and some from other continents came to learn and write papers. Three of them acquired $\mathrm{PhD}$ degrees (Mario Corrales, Ahmad Hatam, Xhemail Barratry). A further 28 Swedish students wrote their $\mathrm{PhD}$ theses supervised by Torgny. He was an inventive and diligent tutor. In a stimulating spirit of comradeship, he offered ample time for counseling and discussing manu- scripts. This often had to take place late in the evenings since his days were filled with routine work, lecturing, planning for research and up-to-date equipment, administration, and so forth. Many times, he characterized the everyday situation in the department as "chaotic," but a fertile chaos it was and for the most part we had great fun and accepted long working days. A typical question from a PhD student late at night was, "May I leave now? I have a relationship with a woman who is still my spouse, you see."

\section{Research and Professional Matters}

Torgny's list of publications includes a wide field of research. Early works dealt with phlebography of the lower extremity and pelvis; others described cerebral vascular anatomy and pathology. In an article from 1954, he and his coauthor Stig Löfstedt, for the first time, suggested that arterial ectasia be defined as a disease entity in its own right, separate from arteriosclerosis, later generally accepted. His interest in contrast media is manifested by several publications-most notably those investigating intrathecal use of metrizamide (Amipaque), the first nonionic contrast medium on the market, synthesized by Nyegaard \& Co in Oslo, Norway. Tests of new products from the x-ray industry (AOT film changer, Mimer III, and the rotating chair) and interventional radiology (nerve root blockade, puncture of spinal cord cysts) were the focus of yet another set of publications.

He excelled in improving established methods as well as inventing new ones: a good example of the latter being his seminal thesis, already mentioned. In this pioneering work, he laid down the principles for determination of regional and global cerebral circulation time that are still followed. Using a rapid film changer (AOT), the circulation time was measured from the angiograms and compared with an isotope method (using iodine 131) devised by himself. The thesis was one of the very first radiologic studies to bring a physiologic dimension into radiology. For the first time, it pointed to the potential of localizing mass lesions by the demonstration of local circulatory disturbances, occasionally even providing improved means for the characterization of lesions. In a series of articles, he further widened the scope of studying cerebral circulation using angiography and the xenon 133 clearance method. He proved that normal pressure hydrocephalus, another special interest to him, was accompanied by a reduced cerebral blood flow and, furthermore, that shunting of the CSF restored the blood flow to a degree that correlated with the clinical improvement. In later years, in cooperation with his disciple and nephew Dan Greitz, Torgny launched an alternative theory for the development of normal pressure hydrocephalus, challenging the bulk-flow theory of CSF. It is based on the assumption that the major cause of this disorder is of vascular origin, more specifically arteriosclerosis of the arteries on the surface of the brain. Although not yet generally accepted, the theory is steadily gaining ground.

In 1976, Torgny Greitz et al published the very first method for stereotactic CT. This work was closely linked to the construction of an all-purpose head-fixation system allowing for exact transfer of positions between imaging modalities, also between imaging and therapeutic modalities (namely, stereotactic surgery and radiation therapy). In 1977, a multidisciplinary PET research project was initiated by Torgny Greitz in cooperation with the Department of Physics at Stockholm University and the Department of 
Neurophysiology at the Karolinska Hospital. A great number of institutions joined in to establish the Stockholm Positron Camera Group, which, before being split up in smaller groups, had its heyday in the 1980s. Torgny's enthusiasm and creativity laid down a fertile ground for cooperation among the many members of the group representing a variety of expertise: neurology, neurosurgery, psychiatry, pharmacology, radio-pharmacy, and so forth. Between 1979 and 1990, Torgny, together with group members, published 47 articles related to PET research; among those, a series of studies focused on metabolic changes in intracranial tumors using various tracers such as glucose and amino acids as well as studies of the use of specific ligands for various neuroreceptors.

Dedicated to studies with PET, Torgny Greitz and coworkers developed a computerized, individually adjustable stereotaxic $3 \mathrm{D}$ brain atlas, based on 0.2-mm-thick histologic cuts. The atlas defined more than 400 structures. It enabled much improved accuracy in defining ROIs in the PET material. It also laid the basis for statistical evaluation at a group level when every single brain in a study had been fitted into a standard anatomy by a set of mathematic transformations. It was a magnificent pioneering work, starting in the early 1980s. Image analysis, according to similar principles, is incorporated in several freeware programs used today.

Torgny shared generously his profound knowledge with the rest of the world by contributing chapters to many books, among those Herbert Abrahams' classic standard work Angiography (first and second editions 1961 and 1971) and the well-known textbooks Radiology of the Skull and Brain and Modern Neuroradiology, both edited by Thomas Newton and Gordon Potts (1981 and 1983). Among his own editorships, The Metabolism of the Human Brain Studied with Positron Emission Tomography stands out as a comprehensive stateof-the-art volume containing the works presented at the 7th Nobel Conference of Karolinska Institutet in 1983.

The Swedish Society of Neuroradiology was founded by Torgny Greitz, Ingmar Wickbom, Erik Lindgren, and Bengt Liliequist in 1971. Torgny took command as acting chairman, 1975-1979, and later contributed regularly in policy discussions related to the advancement of our discipline. As president of the Stockholm Symposium Neuroradiologicum in 1986, he became a prominent profile at the Past Presidents Board. Torgny's generous lecturing as an invited speaker all over the world, though mostly in North America, has been returned when distinguished colleagues have accepted invitations to honor him by coming to Stockholm and giving the "Torgny Greitz Lecture" (instituted by the Swedish Society of Neuroradiology at the time of his retirement); among them were George du Boulay, Giovanni di Chiro, Derek Harwood-Nash, James Barkowich, and Anne Osborne, to just mention a few "giants" in neuroradiology.

\section{Honors}

Torgny Greitz was honored with membership in the Swedish Academy of Science and many radiologic societies, among them the American Society of Neuroradiology, European Society of Neuroradiology, American College of Radiology, International Society of Radiology, Swedish Society of Neuroradiology, Swedish Society of Radiology, Association Francaise de Neuroradiologie, and Nordic Society of Neuroradiology. Among the many prizes he was awarded, he particularly cherished the Ziedses des
Plantes Medaille (1988). In 1985, Torgny was invited by the British Institute of Radiology to give the Mackenzie Davidsson Memorial Lecture.

\section{Personality}

Being head of Sweden's only radiology department exclusively dedicated to neuroradiology, Torgny Greitz was the undisputed leader in our country; the discipline personified. We sometimes called him the "figurehead," of which he disapproved, but, in a way, appropriate considering his tall figure and sharp profile. Torgny had a dynamic leader's capacity to get things done. A telling story is how he managed to acquire the first CT scanner in Europe outside the United Kingdom. In a situation when austerity was proclaimed by the hospital administration, he ordered a scanner from EMI (London, UK), without a cent in his pocket and invited the neuroradiology sections at the other 6 university hospitals in Sweden to join him in writing applications for funding and to participate in the evaluation and development of the CT technique. In October 1973, the scanner was installed in his department, which started a spurt of activity around our country, resulting in a thick supplement of Acta Radiologica (1975), presenting a preliminary assessment of the value of the technique in a number of clinical applications. On December 10, 1979, a triumphant Torgny Greitz would introduce Geoffrey Hounsfield and Allan McCormack at the Nobel Prize ceremony. It was an achievement of masterly rhetoric to convince half the Nobel Assembly at Karolinska Institutet to vote for an engineer and a physicist as laureates of that year's Nobel Prize in Physiology or Medicine. Decided by lot (vote being 50 versus 50 ), the prize was awarded for the invention of Computer-Assisted Tomography, a revolutionary diagnostic tool, certainly a worthy winner, bringing something very useful to mankind and in good agreement with the wording in Alfred Nobel's Will.

Torgny Greitz reached his unique position not only from ambition but essentially by hard work. He was uncompromising in his quest for truth and progress. With his idealistic mind and exceptional persistence, he worked with dedication, addiction as some would say, to improve the methods, the hardware, and software alike, to expand our discipline and to drive it to ever increasing perfection. At the time of presentation, Torgny Greitz's research was foresighted and at the cutting edge, often at the center of discussion in the neuroradiology community even on a broader scale in allied disciplines. Much of his research has been incorporated in the body of today's knowledge of neuroradiology.

His authority was absolute, though his leadership, nonauthoritarian. He got followers by setting a good example for others. With his wit, his boyishness, his humanity, and warm heart, he made friends wherever he set foot. By being a good doctor, an outstanding scientist, and a great innovator, Torgny Greitz is a worthy role model for generations to come.

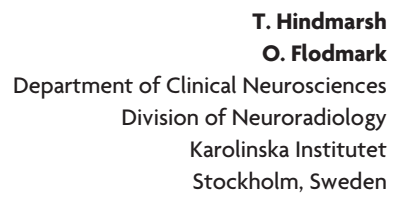

http://dx.doi.org/10.3174/ajnr.A5365 